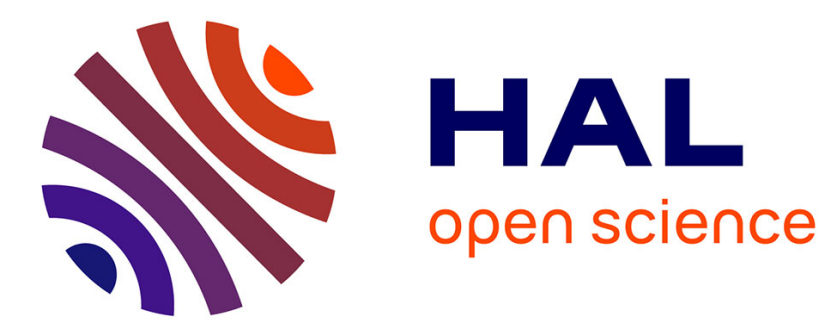

\title{
Contribution à l'étude des boues résiduaires issues de stations d'épuration urbaine dans les plantations forestières
}

B. Ouanouki, S. Igoud

\section{- To cite this version:}

B. Ouanouki, S. Igoud. Contribution à l'étude des boues résiduaires issues de stations d'épuration urbaine dans les plantations forestières. Revue forestière française, 1993, 45 (2), pp.153-158. 10.4267/2042/26406 . hal-03443907

\section{HAL Id: hal-03443907 \\ https://hal.science/hal-03443907}

Submitted on 23 Nov 2021

HAL is a multi-disciplinary open access archive for the deposit and dissemination of scientific research documents, whether they are published or not. The documents may come from teaching and research institutions in France or abroad, or from public or private research centers.
L'archive ouverte pluridisciplinaire HAL, est destinée au dépôt et à la diffusion de documents scientifiques de niveau recherche, publiés ou non, émanant des établissements d'enseignement et de recherche français ou étrangers, des laboratoires publics ou privés. 


\title{
CONTRIBUTION À L'ÉTUDE DES BOUES RÉSIDUAIRES ISSUES DE STATIONS D'ÉPURATION URBAINE DANS LES PLANTATIONS FORESTIĖRES
}

\author{
B. OUANOUKI - S. IGOUD
}

Ces dernières années, un intérēt particulier a été marqué pour les études concernant la revalorisation des sous-produits issus de stations d'épuration urbaine.

Ainsi, cette étude présente les résultats d'une expérimentation d'épandage de boues anaérobies sur sol remanié et concernant deux espèces forestières.

L'aspect positif des épandages de boues résiduaires a été démontré dans de nombreuses études notamment en France et au Canada. Ainsi, au Québec, Couillard (1986), en utilisant de la boue anaérobie liquide, a constaté que des semis de Mélèze "laricina" se comportent d'une manière satisfaisante sur un substrat aussi pauvre que du sable, et que la production de la biomasse croît de $1140 \%$ après quatre mois d'essai en serre.

Au Canada, Gagnon (1972), en appliquant une dose de $560 \mathrm{~kg} / \mathrm{ha}$ d'une boue digérée, a montré qu'une plantation d'Épinette blanche (Picea glauca) présentait, six ans après l'épandage, un gain de croissance en hauteur de près de $40 \%$.

Couillard et Grenier (1988) ont pu noter que la production de biomasse augmentait avec des applications de boues répétées (comparativement à une application massive), et que, pour un même nombre d'applications, les doses plus grandes produisent de meilleurs résultats. Ils ont, en outre, pu mettre en évidence qu'un grand nombre d'applications de petites doses est préférable à un plus petit nombre d'applications de doses plus grandes, les quantités utilisées durant l'expérience variant de $605 \mathrm{~kg} / \mathrm{ha}$ à $10890 \mathrm{~kg} / \mathrm{ha}$ en boues sèches.

Le Tacon et al. (1978) rapportent que l'élimination de la phase de maturation diminue le taux de survie des plantations. Les plants survivants souffrent de nécroses, chloroses et autres symptômes de toxicité; les taux de survie variant de $60 \%$ avec une boue non stabilisée, à $100 \%$ après six mois d'épandage de la boue à l'air libre. Les auteurs ont également relevé la nette augmentation de la biomasse produite et l'amélioration sensible de la nutrition minérale suite à des apports élevés (1000 t/ha).

Le Tacon et al. (1988) ont également mis en relief l'augmentation de la croissance du Frêne planté en intercalaire avec l'Aulne blanc, suite à l'épandage de boues résiduaires.

Le climat particulier de la région et les conditions spécifiques de la conduite des pépinières nous ont semblé être des facteurs intéressants, pouvant induire des comportements positifs quant aux paramètres étudiés. 


\section{MATÉRIEL ET MÉTHODE}

Le dispositif se compose de quatre parcelles de $918 \mathrm{~m}^{2}$ de surface unitaire; chacune d'elle est divisée en six placettes (figure 1 , ci-dessous). La première placette a reçu 2 tonnes de boues ${ }^{(1)}$; la seconde 8 tonnes; la troisième 15 tonnes; la quatrième a été utilisée comme témoin. Au niveau de chaque parcelle, la première placette unitaire est plantée en Pin maritime, la deuxième en Acacia et la troisième en plants alternés de Pin et d'Acacia. Cette disposition est répartie en deux blocs identiques: le premier a été soumis à une irrigation en eau de forage et le deuxième à une irrigation en eau épurée. L'écartement entre les plants a été de $3 \mathrm{~m} \times 3 \mathrm{~m}$.

Figure I

PROTOCOLE EXPÉRIMENTAL

Dose 1: 2 tonnes de boues (144 plants)

Dose 2: 8 tonnes de boues (144 plants)

Dose 3: 15 tonnes de boues (144 plants)

Total (Pins et Acacia) : 576 plants

\begin{tabular}{|c|c|c|c|c|c|}
\hline \multicolumn{3}{|c|}{ TÉMOIN } \\
\hline \multicolumn{3}{|c|}{ EAU DE FORAGE } & \multicolumn{3}{c|}{ EAU ÉPURÉE } \\
\hline Pin & Acacia & $\begin{array}{c}\text { Pin } \\
+ \\
\text { Acacia }\end{array}$ & Pin & Acacia & $\begin{array}{c}\text { Pin } \\
+ \\
\text { Acacia }\end{array}$ \\
\hline
\end{tabular}

\begin{tabular}{|c|c|c|c|c|c|}
\hline \multicolumn{6}{|c|}{ TRAITEMENTS } \\
\hline \multicolumn{3}{|c|}{ EAU DE FORAGE } & \multicolumn{3}{|c|}{ EAU ÉPURÉE } \\
\hline Pin & Acacia & $\begin{array}{c}\text { Pin } \\
+ \\
\text { Acacia }\end{array}$ & Pin & Acacia & $\begin{array}{c}\text { Pin } \\
+ \\
\text { Acacia }\end{array}$ \\
\hline Pin & Acacia & $\begin{array}{c}\text { Pin } \\
+ \\
\text { Acacia }\end{array}$ & Pin & Acacia & $\begin{array}{c}\text { Pin } \\
+ \\
\text { Acacia }\end{array}$ \\
\hline Pin & Acacia & $\begin{array}{c}\text { Pin } \\
+ \\
\text { Acacia }\end{array}$ & Pin & Acacia & $\begin{array}{c}\text { Pin } \\
+ \\
\text { Acacia }\end{array}$ \\
\hline
\end{tabular}

Chaque placette unitaire est plantée de 24 plants disposés en quinconce. Les boues dont la composition est donnée au tableau I (p. 155) ont subi une maturation de 6 mois à l'air libre, avant d'être épandues par cover-crop. Un désherbage mécanique a précédé la plantation qui a été effectuée les 4,5 et 6 février 1991. L'irrigation (10 litres par plant) a été faite en deux fois: les 4 et 6 juin et les 6 et 7 juillet 1991. L'utilisation des eaux épurées (riches en azote et en phosphore) a pour but de connaître leur influence sur les caractéristiques des paramètres étudiés: biomasse, nutrition minérale et croissance en hauteur des plants. Étant donné l'objectif assignè à ces eaux épurées, à savoir leur emploi en agriculture, leur teneur en métaux lourds est supérieure à la norme proposée par Ayers et Wescott (1988).

Le sol de texture argilo-limoneuse se caractérise par son alcalinité $(\mathrm{pH}=7,9$ à 8,4$)$ et sa faible concentration en éléments fertilisants majeurs tels que l'azote, le phosphore et le potassium. Le rapport $\mathrm{C} / \mathrm{N}$ révèle une minéralisation élevée; par contre, le sol est riche en $\mathrm{Ca}$ et $\mathrm{Mg}$. Les concentrations en métaux lourds sont largement inférieures aux concentrations admissibles dans les sols où l'épandage des boues peut s'effectuer (Barideau, 1986).

(1) Il s'agit, dans tous les cas, de boues séchèes à l'air libre pendant six mois. 
Technique et forêt

Tableau I

Résultats d'analyses des boues résiduaires

\begin{tabular}{|c|c|c|c|c|c|}
\hline \multicolumn{2}{|c|}{ Eléments } & Boues fraîches & Boues sèches & \multicolumn{2}{|c|}{ Normes } \\
\hline$N$ & $(\%)$ & 4,610 & 3,820 & \multicolumn{2}{|c|}{$2-2,5(1)$} \\
\hline $\mathrm{P}$ & $(\%)$ & 0,146 & 0,120 & \multicolumn{2}{|c|}{$0,43-0,87(1)$} \\
\hline K & $(\%)$ & 0,090 & 0,066 & \multicolumn{2}{|c|}{$0,16-0,40(1)$} \\
\hline $\mathrm{C}$ & $(\%)$ & 20,60 & 10,27 & \multicolumn{2}{|c|}{ - } \\
\hline Matière organ & $(\%)$ & 35,51 & 17,70 & \multicolumn{2}{|c|}{$40-65(1)$} \\
\hline $\mathrm{C} / \mathrm{N}$ & & 4,46 & - & \multicolumn{2}{|c|}{-} \\
\hline C.E (mmho $\cdot$ & $25^{\circ} \mathrm{C}$ & 0,30 & 0,005 & \multicolumn{2}{|c|}{-} \\
\hline $\mathrm{pH}$ (eau) & & 12,2 & 7,8 & \multicolumn{2}{|c|}{-} \\
\hline Calcaire total & $(\%)$ & 44,36 & 30,20 & \multicolumn{2}{|c|}{ - } \\
\hline Calcaire actif & $(\%)$ & 14,37 & 11,87 & \multicolumn{2}{|c|}{-} \\
\hline $\mathrm{Na}$ & $(\%)$ & 0,04 & 0,02 & \multicolumn{2}{|c|}{ - } \\
\hline $\mathrm{Ca}$ & $(\%)$ & 5,13 & 2,19 & \multicolumn{2}{|c|}{-} \\
\hline $\mathrm{Mg}$ & (ppm) & 4728 & 960 & \multicolumn{2}{|c|}{ - } \\
\hline $\mathrm{Mn}$ & (ppm) & 265 & 189 & \multicolumn{2}{|c|}{$800(1)$} \\
\hline $\mathrm{Fe}$ & (ppm) & 25060 & 6020 & \multicolumn{2}{|c|}{-} \\
\hline $\mathrm{Zn}$ & (ppm) & 2240 & 386 & \multicolumn{2}{|c|}{$3000(1), 2500(2)$} \\
\hline $\mathrm{Cu}$ & (ppm) & 510 & 393 & \multicolumn{2}{|c|}{$1000(1), \quad 600(2)$} \\
\hline $\mathrm{Cr}$ & (ppm) & 70 & 63 & \multicolumn{2}{|c|}{$1000(1), \quad 500(2)$} \\
\hline $\mathrm{Pb}$ & (ppm) & 43,4 & 40,6 & \multicolumn{2}{|c|}{$800(1), \quad 300(2)$} \\
\hline $\mathrm{Cd}$ & (ppm) & 7 & 6,4 & $20(1)$ & $10(2)$ \\
\hline $\mathrm{Hg}$ & (ppm) & 1,36 & 1,03 & $10(1)$ & $5(2)$ \\
\hline
\end{tabular}

(1) Normes d'aprés Lacee (1985).

(2) Concentrations guides, d'après Couillard et Barideau (1990).

\section{PARAMĖTRES MESURÉS}

La croissance en hauteur, la biomasse et la nutrition minérale des deux espèces ont èté les principaux paramètres étudiés.

Mesure de la hauteur. Pour chaque plant, elle a été effectuée à partir du collet jusqu'à l'apex du bourgeon terminal; deux mesures ont été faites: la première le 8 février et la deuxième le 25 juillet 1991.

Taux de survie. II a été approché en multipliant le nombre de plants vivants sur le nombre tota! de plants traduit en pour cent.

Nutrition minérale. Tous les éléments majeurs et oligo-éléments ont été analysés ; deux mesures ont étè prises: la première le 2 février 1991 et la deuxième le 15 juillet 1991.

\section{RÉSULTATS}

Effets des boues sur le taux de survie (tableau II, p. 156)

Après six mois de plantation, le taux de survie est élevé atteignant son maximum de $87,5 \%$ pour l'Acacia avec la dose 3. En moyenne, il se situe autour de $84 \%$ pour les autres traitements. 


\section{B. OUANOUKI - S. IGOUD}

Croissance en hauteur (tableau III, ci-dessous)

En général, les gains de croissance en hauteur augmentent avec des apports plus importants de boues. Le test $F$ est très significatif entre les doses appliquées. Les augmentations de croissance sont maximales pour la dose 3 et décroissent pour les doses 2 et 1 au niveau de tous les traitements. Les meilleurs traitements sont ceux ayant reçu une dose maximale de boues avec des gains de croissance qui sont respectivement de $71,87 \%$, de $68,38 \%$ et de $73 \%$ pour le Pin seul, le Pin en mélange et l'Acacia. L'influence de l'Acacia mélangé au Pin sur la croissance du Pin ne s'est pas manifestée d'une manière conséquente: ce résultat était attendu du fait de la courte période d'expérimentation. L'enrichissement du sol en azote par le biais des nodules n'intervient qu'à long terme.

Tableau II

Taux de survie des plants en fonction des traitements

Taux de survie: (nombre des plants vivants/nombre total des plants) $\times 100$

\begin{tabular}{|c|c|c|c|c|c|c|c|c|c|}
\hline & \multirow{2}{*}{ Espèces } & \multicolumn{2}{|c|}{ Témoin } & \multicolumn{2}{|c|}{ Dose 1} & \multicolumn{2}{|c|}{ Dose 2} & \multicolumn{2}{|c|}{ Dose 3} \\
\hline & & Survie & en $\%$ & Survie & en $\%$ & Survie & en $\%$ & Survie & en $\%$ \\
\hline \multirow{4}{*}{ 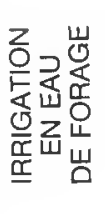 } & Pin $\ldots \ldots \ldots \ldots$ & $15 / 24$ & 62,50 & $20 / 24$ & 83,33 & $15 / 24$ & 62,50 & $12 / 24$ & 50 \\
\hline & Acacia $\ldots . . . \ldots$ & $18 / 24$ & 75 & $22 / 24$ & 91,66 & $17 / 24$ & 70,93 & $20 / 24$ & 83,33 \\
\hline & Pin (en mélange) . . . . . & $8 / 12$ & 66,66 & $8 / 12$ & 66 & $7 / 12$ & 58,33 & $9 / 12$ & 66,66 \\
\hline & Acacia (en mélange) ... & $8 / 12$ & 66,66 & $10 / 12$ & 83,33 & $8 / 12$ & 66,66 & $9 / 12$ & 75 \\
\hline \multirow{4}{*}{ 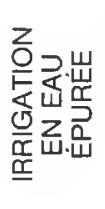 } & Pin $\ldots \ldots \ldots$ & $19 / 24$ & 79,16 & $20 / 24$ & 83,33 & $18 / 24$ & 75 & $19 / 24$ & 79,16 \\
\hline & Acacia .......... & $20 / 24$ & 83,33 & $21 / 24$ & 87,50 & $20 / 24$ & 83,33 & $21 / 24$ & 87,50 \\
\hline & Pin (en mélange) & $9 / 12$ & 75 & $9 / 12$ & 75 & $8 / 12$ & 66,66 & $8 / 12$ & 75 \\
\hline & Acacia (en mélange) .. & $10 / 12$ & 93,83 & $10 / 12$ & 83,33 & $9 / 12$ & 75 & $18 / 12$ & 83,33 \\
\hline
\end{tabular}

Dose 1: 21,76 tonnes / hectare de boues.

Dose 2: 87,14 tonnes / hectare de boues. Dose $3: 163,40$ tonnes / hectare de boues.

Tableau III Moyennes des gains de croissance en fonction des doses de boues après 6 mois d'expérimentation

Moyenne des gains de croissance: en $\mathrm{cm}: \sum_{1-1}^{n} \frac{\mathrm{hi}}{\mathrm{n}}$ en $\%=\frac{F_{D}-F_{\mathrm{T}}}{F_{\mathrm{T}}} \times 100$

$F_{D}=$ Hauteur moyenne des gains de croissance; des traitements

$\mathrm{F}_{\mathrm{T}}=$ Hauteur moyenne des gains de croissance; du témoin

\begin{tabular}{|c|c|c|c|c|c|c|c|c|}
\hline & \multirow{2}{*}{ Espèces } & \multirow{2}{*}{$\begin{array}{c}\text { Témoin } \\
\mathrm{cm}\end{array}$} & \multicolumn{2}{|c|}{ Dose 1} & \multicolumn{2}{|c|}{ Dose 2} & \multicolumn{2}{|c|}{ Dose 3} \\
\hline & & & $\mathrm{cm}$ & $\%$ & $\mathrm{~cm}$ & $\%$ & $\mathrm{~cm}$ & $\%$ \\
\hline \multirow{4}{*}{ 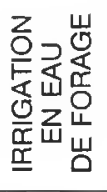 } & Pin $\ldots \ldots \ldots \ldots$ & 7,04 & 7,32 & 3,97 & 8,64 & 22,72 & 12,10 & 71,87 \\
\hline & Acacia & 7,96 & 9,09 & 14,19 & 12,62 & 58,54 & 13,77 & 72,98 \\
\hline & Pin (en mélange) & 6,61 & 4,81 & 0 & 8,97 & 34,68 & 7,63 & 14,65 \\
\hline & Acacia (en mélange) & 5,24 & 7,05 & 0 & 12,30 & 49,27 & 13,91 & 66,98 \\
\hline \multirow{4}{*}{ 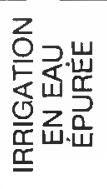 } & Pin $\ldots \ldots \ldots \ldots$ & 7,06 & 7,98 & 13,03 & 11,87 & 68,13 & 12,11 & 71,51 \\
\hline & Acacia & 8,38 & 8,59 & 2,50 & 12,25 & 46,18 & 13,58 & 62,05 \\
\hline & Pin (en mélange) . . . . . & 6,61 & 8,54 & 7,86 & 8,83 & 33,58 & 11,13 & 68,38 \\
\hline & Acacia (en mèlange) & 8,34 & 8,58 & 2,52 & 8,60 & 5,64 & 13,21 & 60,31 \\
\hline
\end{tabular}

Dose 1: 21,78 tonnes/hectare de boues.

Dose 2 : 87,14 tonnes / hectare de boues.

Dose $3: 163,40$ tonnes / hectare de boues. 


\section{Technique et forêt}

Nutrition minérale (tableaux IV et $\mathrm{V}$, ci-dessous)

Les analyses foliaires du Pin ont montré qu'il n'existait pas de carence pour l'azote et le potassium, mais que le phosphore se situe à un niveau critique; par contre, l'Acacia présente une carence en phosphore et en potassium. Pour les autres éléments, leur concentration est considérée comme normale.

Tableau IV Résultats d'analyses foliaires de Pinus pinaster après traitement

\begin{tabular}{|c|c|c|c|c|c|c|c|c|c|c|c|}
\hline \multicolumn{2}{|c|}{$\begin{array}{l}\text { Teneurs des éléments minéraux } \\
\text { dans les aiguilles de Pin }\end{array}$} & $\begin{array}{c}N \\
(\%)\end{array}$ & $\begin{array}{l}P \\
(\%)\end{array}$ & $\begin{array}{c}K \\
(\%)\end{array}$ & $\begin{array}{l}\mathrm{Na} \\
(\%)\end{array}$ & $\begin{array}{l}\mathrm{Ca} \\
(\%)\end{array}$ & $\begin{array}{l}\mathrm{Mg} \\
(\%)\end{array}$ & $\begin{array}{c}\mathrm{Mn} \\
(\mathrm{ppm})\end{array}$ & $\begin{array}{c}\mathrm{Fe} \\
(\mathrm{ppm})\end{array}$ & $\underset{(\mathrm{ppm})}{\mathrm{Zn}}$ & $\begin{array}{c}\mathrm{Cu} \\
\text { (ppm) }\end{array}$ \\
\hline \multirow{8}{*}{ 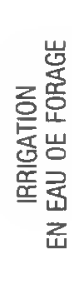 } & Témoin & 1,03 & 0,03 & 0,45 & 1,67 & 0,20 & 0,21 & 56,00 & 43,40 & 25,65 & 10,35 \\
\hline & Dose 1 & 1,15 & 0,08 & 0,70 & 1,30 & 0,49 & 0,16 & 51,35 & 37,66 & 50,23 & 44,82 \\
\hline & Dose 2 & 1,20 & 0,18 & 0,80 & 1,46 & 1,05 & 0,20 & 63,27 & 46,00 & 40,56 & 25,45 \\
\hline & Dose 3 & 1,52 & 0,15 & 1,10 & 1,20 & 1,19 & 0,22 & 66,72 & 57,26 & 113,86 & 16,22 \\
\hline & Témoin (en mélange) . . & 1,39 & 0,03 & 0,65 & 1,10 & 0,14 & 0,15 & 31,06 & 39,30 & 34,03 & 11,30 \\
\hline & Dose 1 (en mélange) & 1,10 & 0,08 & 0,65 & 1,51 & 0,68 & 0,18 & 40,74 & 50,86 & 64,84 & 15,53 \\
\hline & Dose 2 (en mélange) & 1,33 & 0,14 & 0,93 & 1,42 & 1,06 & 0,19 & 74,34 & 75,43 & 57,40 & 12,25 \\
\hline & Dose 3 (e & 1,76 & 0,20 & 1,02 & 1,67 & 1,18 & 0,23 & 72,20 & 76,73 & 102,31 & 12,90 \\
\hline \multirow{8}{*}{ 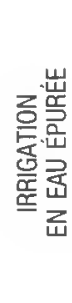 } & noi & 1,07 & 0,04 & 0,64 & 1. & 0 , & 0 , & 44,44 & 59,89 & 25,63 & 10,20 \\
\hline & Dose $1 \ldots$ & 1,14 & 0,11 & 0,66 & 1,34 & 0,60 & 0,21 & 36,26 & 65,93 & 36,23 & 8,06 \\
\hline & Dose 2 & 1,25 & 0,16 & 0,83 & 1,60 & 0,81 & 0,24 & 58,63 & 61,80 & 28,60 & 19,20 \\
\hline & Dose 3 & 1,38 & 0,16 & 0,85 & 1,74 & 1,21 & 0,21 & 49,13 & 114,03 & 36,63 & Traces \\
\hline & Témoin (en mẻlange) & 1,06 & 0,05 & 0,57 & 1,18 & 0,20 & 0,22 & 50,93 & 59,33 & 55,33 & 8,46 \\
\hline & Dose 1 (en mėlange) ... & 1,19 & 0,13 & 0,74 & 1,25 & 0,37 & 0,17 & 46,43 & 78,86 & 51,41 & 20,43 \\
\hline & Dose 2 (en mélange) . & 1,32 & 0,13 & 0,75 & 1,30 & 0,78 & 0,2 & 80,06 & 93,00 & 74,60 & 14,73 \\
\hline & Dose 3 (en mélange). & 1,45 & 0,19 & 0,77 & 2,44 & 1,09 & 0,18 & 81,56 & 114,00 & 72,23 & 10,30 \\
\hline
\end{tabular}

Tableau $\mathrm{V}$

Résultats d'analyses foliaires d'Acacia cyanophylla après traitement

\begin{tabular}{|c|c|c|c|c|c|c|c|c|c|c|c|}
\hline \multicolumn{2}{|c|}{$\begin{array}{l}\text { Teneurs des éléments minéraux } \\
\text { dans les feuilles d'Acacia }\end{array}$} & $\begin{array}{c}N \\
(\%)\end{array}$ & $\begin{array}{c}P \\
(\%)\end{array}$ & $\begin{array}{c}K \\
(\%)\end{array}$ & $\begin{array}{l}\mathrm{Na} \\
(\%)\end{array}$ & $\begin{array}{l}\mathrm{Ca} \\
(\%)\end{array}$ & $\begin{array}{l}\mathrm{Mg} \\
(\%)\end{array}$ & $\begin{array}{c}\mathrm{Mn} \\
(\mathrm{ppm})\end{array}$ & $\begin{array}{c}\mathrm{Fe} \\
(\mathrm{ppm})\end{array}$ & $\underset{(\mathrm{ppm})}{\mathrm{Zn}}$ & $\underset{(\mathrm{ppm})}{\mathrm{Cu}}$ \\
\hline \multirow{8}{*}{ 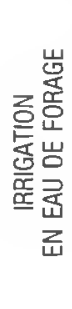 } & Témoin & 1,84 & 0,09 & 0,58 & 1,32 & 1,52 & 0,23 & 79,73 & 67,16 & 52,20 & 17,40 \\
\hline & Dose 1 & 1,86 & 0,16 & 0,60 & 1,36 & 0,58 & 0,16 & 51,26 & 27,26 & 46,40 & 22,06 \\
\hline & Dose 2. & 2,02 & 0,17 & 0,62 & 1,26 & 0,75 & 0,20 & 34,73 & 72,83 & 70,33 & 10,77 \\
\hline & Dose 3 & 2,46 & 0,29 & 0,82 & 1,35 & 1,61 & 0,27 & 37,26 & 107,00 & 55,96 & 12,82 \\
\hline & Tèmoin (en mélange) & 1,52 & 0,04 & 0,79 & 1,31 & 0,50 & 0,11 & 31,09 & 83,83 & 39,16 & 31,00 \\
\hline & Dose 1 (en mélange) & 1,69 & 0,13 & 0,68 & 1,02 & 0,73 & 0,19 & 49,86 & 101,90 & 41,30 & 15,60 \\
\hline & Dose 2 (en mélange) & 2,00 & 0,15 & 0,54 & 1,19 & 0,37 & 0,28 & 32,80 & 42,96 & 51,76 & 15,00 \\
\hline & Dose 3 (en mélange) . . & 2,20 & 0,19 & 0,76 & 1,31 & 1,83 & 0,27 & 37,60 & 112,50 & 56,63 & 13,80 \\
\hline \multirow{8}{*}{ 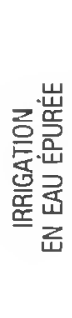 } & IElliuin & 1,56 & 0,07 & 0,56 & 1,32 & 0,48 & 0,20 & 50,95 & 84,85 & 77,63 & 19,60 \\
\hline & Dose 1 & 2,21 & 0,13 & 0,81 & 1,29 & 0,62 & 0,19 & 47,43 & 29,03 & 45,43 & 21,50 \\
\hline & Dose 2 & 2,37 & 0,19 & 0,83 & 1,22 & 0,76 & 0,16 & 37,70 & 84,80 & 65,36 & 31,00 \\
\hline & Dose 3 & 2,81 & 0,22 & 1,26 & 1,36 & 1,76 & 0,25 & 40,13 & 122,06 & 78,76 & Traces \\
\hline & Tèmoin (en mélange) ... & 1,64 & 0,04 & 0,57 & 1,31 & 0,54 & 0,21 & 58,08 & 26,53 & 46,53 & 28,88 \\
\hline & Dose 1 (en mélange) ... & 2,01 & 0,09 & 0,67 & 1,07 & 0,67 & 0,19 & 50,36 & 118,23 & 39,16 & 17,46 \\
\hline & Dose 2 (en mélange) & 2,19 & 0,15 & 0,83 & 1,19 & 0,72 & 0,28 & 32,60 & 126,93 & 46,33 & 8,33 \\
\hline & Dose 3 (en mélange) & 2,42 & 0,22 & 0,95 & 1,36 & 1,86 & 0,27 & 69,36 & 59,06 & 64,66 & 10,10 \\
\hline
\end{tabular}


Ces valeurs n'ont valeur que d'ordre général, car elles fluctuent au gré des saisons et de la période de prélèvement.

La concentration en $\mathrm{N}, \mathrm{P}, \mathrm{Na}$ et en $\mathrm{Zn}$ augmente avec la dose de boue, atteignant même une consommation de luxe pour le $\mathrm{P}$ : sa teneur évolue d'une manière positive quand les apports de boues sont croissants.

Les boues en question, quoique pauvres en $\mathrm{P}$, font que les fortes doses appliquées, la faible tendance de l'élément $P$ au lessivage, et sa forme assimilable dans l'eau épurée, ont favorisé son absorption racinaire.

Pour les autres éléments tels $\mathrm{Fe}, \mathrm{Mg}$, Ca et $\mathrm{Cu}$, leur concentration augmente quand les apports de boues se font plus importants; le test $F$ est alors non significatif.

Pour les métaux lourds, leur état sous forme de traces seulement est dû certainement au $\mathrm{pH}$ alcalin qui empêche leur absorption racinaire.

\section{CONCLUSIONS}

Les résultats de l'expérimentation ainsi menée vont dans le même sens que les essais rapportés en bibliographie, où l'apport des boues anaérobies a favorisé la croissance en hauteur, donc une augmentation en rendement de produits ligneux; le taux de survie s'est trouvé également amélioré grâce notamment à une meilleure nutrition en éléments minéraux. Cependant, on n'a pas pu mettre en évidence un rôle particulier de l'espèce fixatrice d'azote qu'est l'Acacia sur les rendements du Pin maritime. Les gains de croissance en hauteur et en éléments nutritifs n'ont pas fait apparaître un rôle significatif de l'eau épurée par rapport à l'eau de forage.

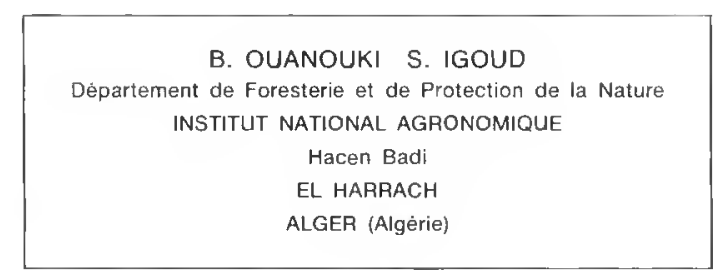

\section{BIBLIOGRAPHIE}

AYERS (R.S.), WESCOTT (D.W.). - Qualité de l'eau en agriculture. - Bulletin FAO d'irrigation et de drainage, $n^{\circ} 29,1988,180 \mathrm{p}$.

BARIDEAU (L.). - Les Boues d'épuration, menace pour l'environnement ou matière première pour l'agriculture? - Bulletin de la Recherche agronomique, 1986, pp. 369-382.

COUILLARD (C.). - Étude de quelques indices de croissance de Larix laricina fertilisés par des boues anaérobies. - Pub. div. Selper Ltd, 1988, pp. 191-206.

COUILLARD (C.), GRENIER (Y.). - Alternative à la gestion des boues résiduaires municipales : recyclage en sylviculture. - Sci. Techn. de l'eau, vol, $20, n^{\circ} 3,1988, p p .215-220$.

GAGNON (J.-D.). - Les Égouts domestiques: un engrais valable en foresterie. - Environnement Canada. Centre de recherches forestières des Laurentides. Québec. Rapport Q-F-X-38, 1972. - 24 P.

LACEE (C.). - Analyses des boues. - AFEE, 1985. - Tome 1, 135 p. Tome 2, 127 p.

LE TACON (F.) et al. - Augmentation de la croissance initiale du Frêne par épandage de boues de station d'épuration urbaine et plantation intercalaire d'Aulne blanc. - Revue forestiére française, vol. $X L, n^{\circ} 2$, 1988, pp. 117-125.

LE TACON (F.) et al. - Valorisation des boues résiduaires de la station d'épuration urbaine en sylviculture. Conséquences sur l'environnement. - INP-Lorraine, 1978. - $44 \mathrm{p}$. 